\title{
Nonlinear dynamic characteristics of SMA gripper under bounded noise
}

\author{
Xin-miao $\mathrm{Li}^{1}$, Zhi-wen $\mathrm{Zhu}^{2}$, and Qing-xin Zhang ${ }^{1, *}$ \\ ${ }^{1}$ Beijing institute of structure and environment engineering, 1 Nan Da Hong Men Road, Beijing \\ 100076, P.R.China \\ ${ }^{2}$ Tianjin key laboratory of nonlinear dynamics and control, 92 Weijin Road, Tianjin 300072, \\ P.R.China
}

\begin{abstract}
A kind of constitutive model of SMA is proposed in this paper, and the nonlinear dynamic response of a SMA gripper under bounded noise is studied. The harmonic driving signals and the random disturbance made up of bounded noise. The dynamic model of the system is established by Hamilton principle. The numerical and experimental results show that there is stochastic resonance in the system; the system's vibration amplitude reaches the most when the outside excitation is moderate.
\end{abstract}

\section{Introduction}

Shape memory alloy (SMA) is a type of smart material, which has shape memory effect. SMA gripper is used in medical field widely [1]. To enhance the accuracy of SMA gripper, its dynamic characteristics should be studied. Many researchers have studied SMA gripper [1-7]. Kohl et al. studied a SMA gripper's dynamic response and control [2]. Just et al. applied position control to a SMA gripper and obtained high control accuracy [3]. To SMA materials, Graesser et al. proposed a three-dimensional SMA constitutive model [4]. Ivshin et al. developed a SMA thermo-mechanical model [5]. Although many achievements have been reported, most of them focused on the constitutive model, and the results of dynamic response of SMA gripper are absent.

SMA gripper used in medicine are controlled by harmonic currents to achieve the opening and closing action. However, SMA gripper are usually under stochastic excitation in the working process. Although the stochastic excitation is weak, it will affect the gripper's motion. The harmonic control force and the stochastic excitation generate a bounded noise, which cause the different dynamic characteristics from the harmonic system.

\footnotetext{
* Corresponding author: zhqx@spacechina.com
} 


\section{SMA constitutive model}

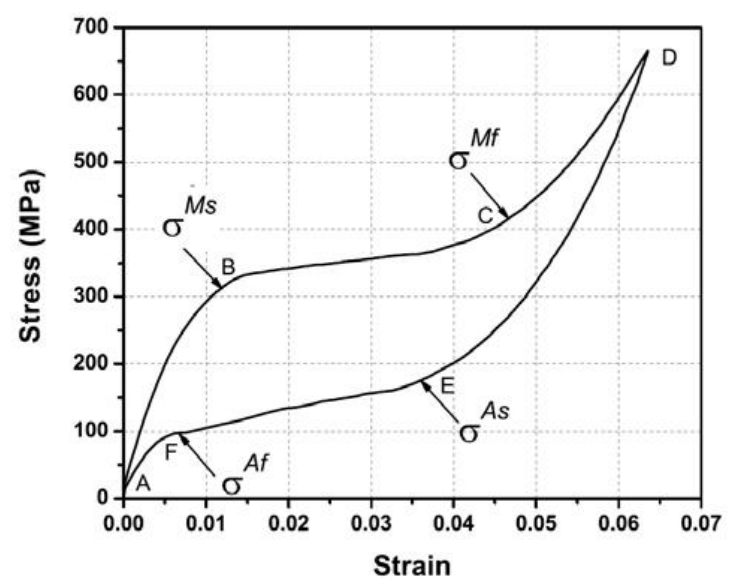

Fig. 1. Strain-stress curves of the SMA.

The experimental results of SMA are shown in Figure 1. The length of Ti-Ni SMA film is $7 \mathrm{~mm}$, the width is $1.5 \mathrm{~mm}$, and the thickness is $0.1 \mathrm{~mm}$. The SMA's austenite finish temperature is $34^{\circ} \mathrm{C}$. Thus, the hysteretic phenomenon is induced by the superelastic behavior of SMA. Zhu et al. established a SMA's constitutive model as follows [8]:

$$
\sigma=\sigma_{1}+\sigma_{2}=a_{1} \varepsilon+a_{2} \varepsilon^{2}+a_{3} \varepsilon^{3}+\left(a_{4} \varepsilon+a_{5} \varepsilon^{2}+a_{6} \varepsilon^{3}+a_{7} \varepsilon^{4}\right) \dot{\varepsilon}
$$

where $\sigma$ is the stress, $\varepsilon$ is the strain. To SMA shown in Figure $1, a_{1}=10000, a_{2}=-32$, $a_{3}=5.7$.

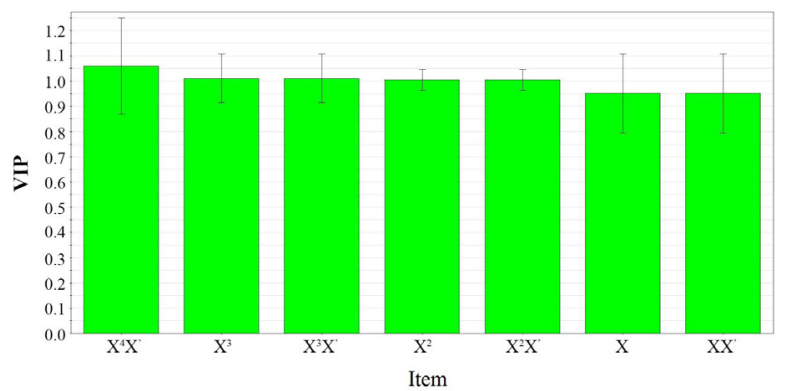

Fig. 2. Variable importance of each term.

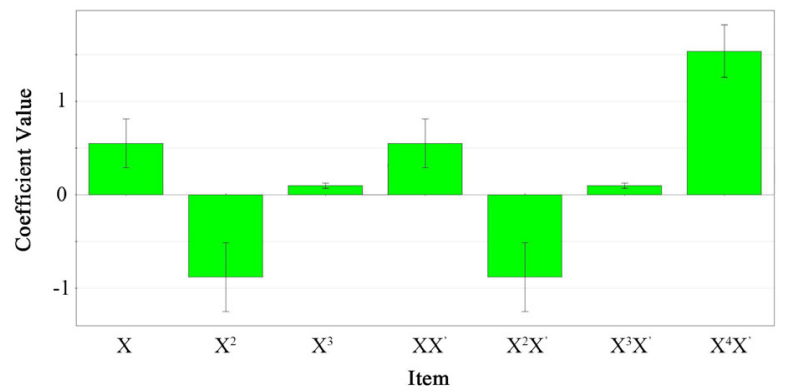

Fig. 3. Coefficient values of each term. 


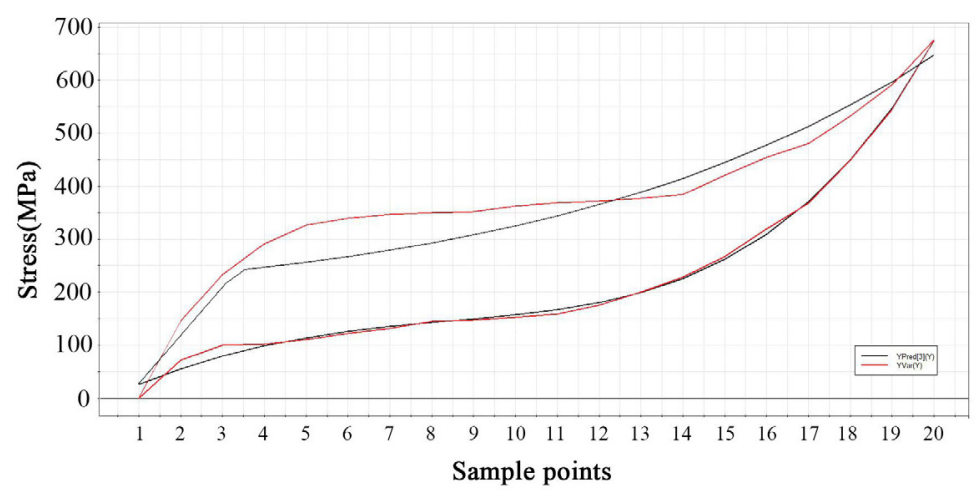

Fig. 4. Results of forecast test for the fitting effect of Eq. (1) on strain-stress data of SMA.

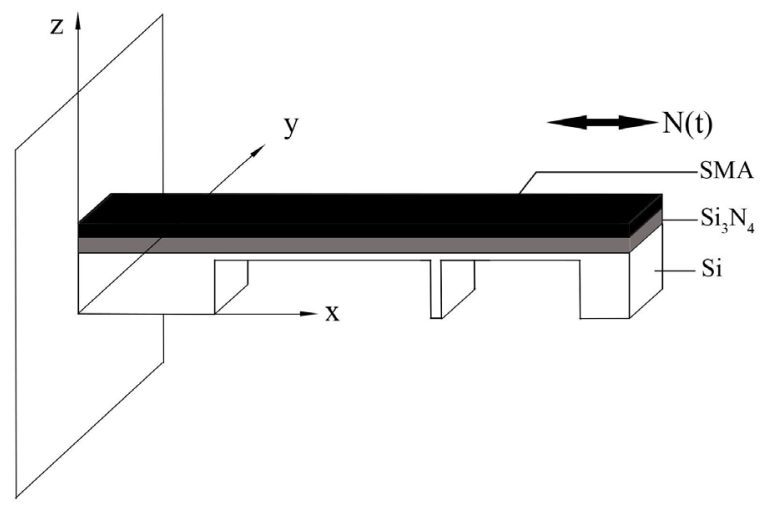

Fig. 5. Results of forecast test for the fitting effect of Eq. (1) on strain-stress data of SMA.

The results of prediction test to Eq. (1) are shown in Figure 4, and the mechanical model of a SMA gripper under bounded noise is shown in Figure 5, where $N(t)=F \sin (\Omega t+\chi+\sigma B(t))$ is the bounded noise. The Hamilton's variational principle is:

$$
\delta \mathrm{S}=\int_{\mathrm{t}_{1}}^{\mathrm{t}_{2}} \delta\left(T_{1}+T_{2}-U_{1}-U_{2}+W_{d}+W\right) d t=0
$$

where

$$
T_{1}=\frac{1}{2} \int_{0}^{L} \rho_{1} A_{1}\left(\frac{\partial u}{\partial t}\right)^{2} d x \quad, \quad T_{2}=\frac{1}{2} \int_{0}^{L} \rho_{2} A_{2}\left(\frac{\partial u}{\partial t}\right)^{2} d x
$$

$U_{1}=\int_{0}^{L} \frac{E_{1} I_{2}}{2}\left(\frac{\partial^{2} u}{\partial x^{2}}\right)^{2} d x+\frac{E_{1} A_{1}}{8 L}\left[\int_{0}^{L}\left(\frac{\partial u}{\partial x}\right)^{2} d x\right]^{2} \quad, \quad U_{2}=\frac{1}{2} E_{2} A_{2} \int_{0}^{\mathrm{L}}\left(\frac{\partial u}{\partial x}\right)^{2} d x$

$W_{d}=-\int_{0}^{L} c u \frac{\partial u}{\partial t} d x, W=\int_{0}^{L} \delta u N d x$.

Thus, the dynamic model of a SMA gripper is: 


$$
\begin{aligned}
& m \frac{\partial^{2} u}{\partial t^{2}}+\left[c+\int_{0}^{L} E_{1} A_{1}\left(a_{4} u+a_{5} u^{2}+a_{6} u^{3}+a_{7} u^{4}\right) d x\right] \frac{\partial u}{\partial t}+b_{1} \frac{\partial^{2} u}{\partial x^{2}} \\
& +b_{2} \frac{\partial^{4} u}{\partial x^{4}}-b_{3} \frac{\partial^{2} u}{\partial x^{2}} \int_{0}^{L}\left(\frac{\partial u}{\partial x}\right)^{2} d x=N+\frac{1}{2}\left(E_{2} A_{2} \frac{\partial^{2} u}{\partial x^{2}}+\rho_{2} A_{2} \frac{\partial^{2} u}{\partial t^{2}}\right)
\end{aligned}
$$

where $m=\frac{\rho_{1} A_{1} l}{L}+\rho_{2} A_{2}, b_{1}=\frac{a_{1} E_{2} A_{2}}{3}+\frac{\varepsilon_{33}^{s} E_{3} A_{1}}{2}, b_{2}=a_{2} E_{2} I_{2}, b_{3}=\frac{E_{1} A_{1}}{8 L}$.

The equation of the system's response are:

$$
\left\{\begin{array}{l}
\ddot{u}_{1}+b_{1} u_{1}+b_{2} u_{1}^{3}+\mu_{1} u_{2}^{2}+\left(2 \eta+b_{3} u_{1}^{2}+b_{4} u_{1}^{4}\right) \dot{u}_{1}=e \sin (\Omega t+\theta+\sigma B(t)) \\
\ddot{u}_{2}+c_{1} u_{1}+c_{2} u_{1}^{3}+\mu_{1} u_{2}^{2}+\left(2 \eta+c_{3} u_{1}^{2}+c_{4} u_{1}^{4}\right) \dot{u}_{1}=g \sin (\Omega t+\theta+\sigma B(t))
\end{array}\right.
$$

where $\eta \quad$ is the damping coefficient, $\quad b_{1}=\frac{a_{1} \pi^{2}\left(\frac{9}{a^{2}}+\frac{1}{b^{2}}\right)^{2}-\frac{\pi^{2}}{b^{2}} N_{0}}{\rho h}$, $c_{1}=\frac{a_{1} \pi^{2}\left(\frac{1}{a^{2}}+\frac{9}{b^{2}}\right)^{2}-\frac{\pi^{2}}{a^{2}} N_{0}}{\rho h}, \quad b_{2}=\frac{a_{3} \pi^{4}}{4 \rho h a b}\left(\frac{27 a}{b^{3}}+\frac{b}{a^{3}}\right), \quad c_{2}=\frac{a_{3} \pi^{4}}{4 \rho h a b}\left(\frac{a}{b^{3}}+\frac{27 b}{a^{3}}\right)$, $b_{3}=\frac{a_{5} \pi^{6}}{16 \rho h a b}\left(\frac{81 a}{b^{4}}+\frac{b}{a^{4}}\right), \quad c_{3}=\frac{a_{5} \pi^{6}}{16 \rho h a b}\left(\frac{a}{b^{4}}+\frac{81 b}{a^{4}}\right), \quad b_{4}=\frac{a_{7} \pi^{8}}{64 \rho h a b}\left(\frac{243 a}{b^{4}}+\frac{b}{a^{4}}\right)$, $c_{4}=\frac{a_{7} \pi^{8}}{64 \rho h a b}\left(\frac{a}{b^{4}}+\frac{243 b}{a^{4}}\right), e=\frac{F \pi^{2}\left(\frac{9}{a^{2}}+\frac{1}{b^{2}}\right)^{2}}{\rho h}, g=\frac{F \pi^{2}\left(\frac{1}{a^{2}}+\frac{9}{b^{2}}\right)^{2}}{\rho h} ; \gamma \quad$ is the coupling coefficient.

Let $u_{1}=q$, the first equation of Eqs.5 can be shown as follows:

$$
\ddot{q}+b_{1} q+b_{2} q^{3}+\left(2 \eta+b_{3} q^{2}+b_{4} q^{4}\right) \dot{q}=e \sin (\Omega t+\theta+\sigma B(t))
$$

\section{Nonlinear dynamic characteristics of a SMA gripper}

When the noise intensity $\sigma=0$, the outside excitation becomes harmonic excitation, and the dynamic model can be shown as follows:

$$
\ddot{q}+b_{1} q+b_{2} q^{3}+\left(2 \eta+b_{3} q^{2}+b_{4} q^{4}\right) \dot{q}=e \sin (\Omega t+\theta)
$$

The solution of Eq. (7) is:

$$
\begin{aligned}
& q=q_{0} \cos (\omega t+\theta)+q_{1} \cos (\omega t+\theta)+q_{2} \cos 3(\omega t+\theta)+ \\
& q_{3} \sin (\omega t+\theta)+q_{4} \sin 3(\omega t+\theta)+q_{5} \cos 5(\omega t+\theta)
\end{aligned}
$$

where, $q_{0}=\frac{e}{\bar{\omega}} b_{1}, q_{1}=\frac{3 e^{3}}{4 \bar{\omega}} b_{2}, q_{2}=-\frac{e^{3}}{4 \bar{\omega}} b_{2}, q_{3}=\frac{3 e^{3}}{4 \bar{\omega}} b_{3} \omega, q_{4}=\frac{e^{3}}{4 \bar{\omega}} b_{3} \omega$, $q_{5}=\frac{e^{5}}{16 \bar{\omega}} b_{4} \omega, \omega=\sqrt{b_{1}}, \bar{\omega}=\sqrt{\left(\Omega^{2}-\omega^{2}\right)^{2}+(2 \eta \omega)^{2}}$.

When the noise intensity $\sigma \neq 0$, the outside excitation becomes bounded noise, and Eq. (6) becomes a stochastic nonlinear differential equation. The averaged Ito equation of Eq. (6) are: 


$$
\left\{\begin{array}{l}
d A=m_{1}\left(A, \Delta^{\prime}\right) d t \\
d \Delta^{\prime}=m_{2}\left(A, \Delta^{\prime}\right) d t+\sigma d B(t)
\end{array}\right.
$$

where

$$
\begin{aligned}
& \Delta^{\prime}=\Omega t+\sigma B(t)+\chi-\Theta \\
& m_{1}\left(A, \Delta^{\prime}\right)=-\pi\left(b_{3} A^{2}+\frac{1}{4} b_{4} A^{4}\right)-\frac{e A}{2 \eta} \cos \Delta^{\prime} \\
& m_{2}\left(A, \Delta^{\prime}\right)=2 \pi \Omega-\frac{1}{\eta}\left(\frac{3}{4} \pi b_{2} A^{3}-\frac{e}{2} \sin \Delta^{\prime}\right)
\end{aligned}
$$

The system's averaged equation is:

$$
\frac{\partial f}{\partial t}=-\frac{\partial}{\partial A}\left(m_{1} f\right)-\frac{\partial}{\partial \Delta^{\prime}}\left(m_{2} f\right)+\frac{\sigma^{2}}{2} \frac{\partial^{2} f}{\partial \Delta^{\prime 2}}
$$

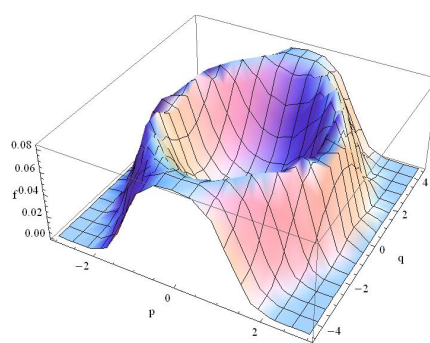

(a) $\sigma=0.1$

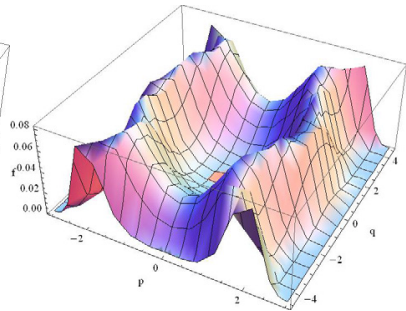

(b) $\sigma=0.5$

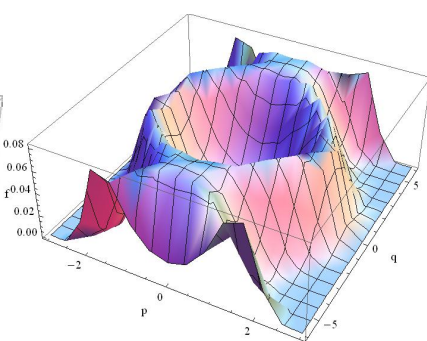

(c) $\sigma=0.8$

Fig. 6. Stationary probability density of the system's response.

The numerical results of the system's response are presented in Figure 6, and the experimental results of SMA gripper under bounded noise are shown in Figures 7-9, where the frequency $\Omega=30 \mathrm{~Hz}$. Ti-Ni alloy is chosen as SMA film. The length of the micro gripper is $10 \mathrm{~cm}$, and its width is $1 \mathrm{~cm}$. The length of SMA thin film is $3 \mathrm{~cm}$, its width is $1 \mathrm{~cm}$, and its thickness is $0.4 \mathrm{~mm}$. The stochastic resonance phenomenon occurs in the process.

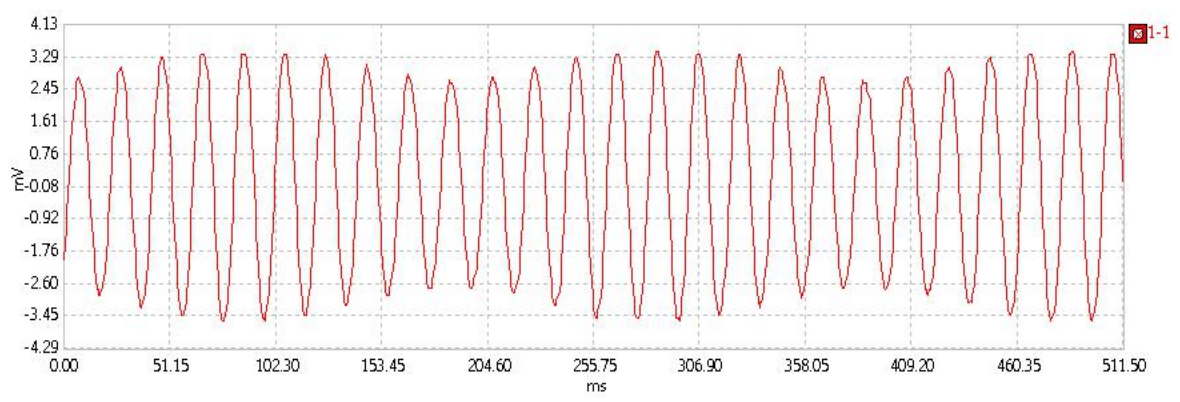

Fig. 7. Response of SMA gripper when $\sigma=0.1$. 


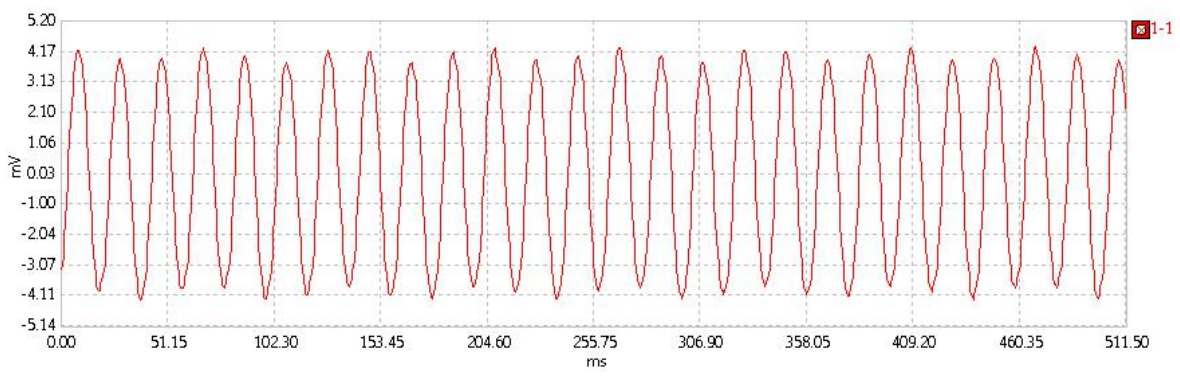

Fig. 7. Response of SMA gripper when $\sigma=0.5$.

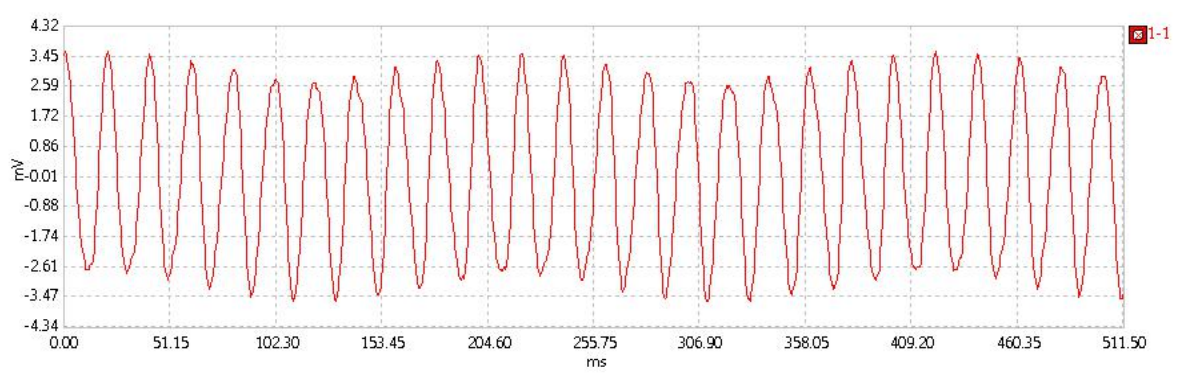

Fig. 7. Response of SMA gripper when $\sigma=0.8$.

\section{Conclusion}

A kind of constitutive model of SMA is proposed in this paper, and the nonlinear dynamic response of a SMA gripper under bounded noise is studied. The harmonic driving signals and the random disturbance made up of bounded noise. The dynamic model of the system is established by Hamilton principle. The numerical and experimental results show that there is stochastic resonance in the system; the system's vibration amplitude reaches the most when the outside excitation is moderate.

\section{Acknowledgements}

The authors gratefully acknowledge the support of NSFC through Grant Nos. 11872266 and 51875396, Chinese Aviation Science Foundation through Grant No. 2016ZA48001, Tianjin RPAFAT through Grant No. 16JCYBJC18800, and Cast-Bisee 511 Program through Grant No. CAST-BISEE2017-006.

\section{References}

[1] B. Winzek, S. Schmitz, and H. Rumpf. Materials Science and Engineering A 3 (2004),

[2] M. Kohl, B. Krevet, and E. Just. Sensors and Actuators A-Physical 2 (2001).

[3] E. Just, M. Kohl, and S. Miyazaki. Journal de Physique IV 8 (2001).

[4] E.J. Graesser and F.A. Cozzarelli. Journal of Intelligent Material Systems and Structures 5 (1994). 
[5] Y. Ivshin and T.J. Pence. Journal of Intelligent Material Systems and Structures 5 (1994).

[6] Z.W. Zhu, Q.X. Zhang and J. Xu. Thin Solid Films 4 (2014). 